\title{
Technical note: Development and evaluation of a standard operating procedure for ultrasound-based measurements of teat canal dimensions in dairy cows
}

\author{
M. Wieland, ${ }^{* 1}$ J. M. Melvin, $†$ P. D. Virkler, ${ }^{*}$ D. V. Nydam, ${ }^{*}$ and W. Heuwieser ${ }^{\star} \ddagger$ \\ *Department of Population Medicine and Diagnostic Sciences, and \\ †Department of Animal Science, Cornell University, Ithaca, NY 14853 \\ ‡Clinic for Animal Reproduction, Faculty of Veterinary Medicine, Freie Universität Berlin, Königsweg 65, 14163 Berlin, Germany
}

\begin{abstract}
The objectives were to (1) develop a standard operating procedure (SOP) for ultrasound-based measurements of teat canal dimensions and (2) determine the precision of ultrasound-based measurements of teat canal dimensions in dairy cows. Teat scans $(\mathrm{n}=64)$ of the right hind and left front teats from 16 cows were obtained with a portable ultrasound device before and after machine milking. Teat dimensions measured were teat canal length (TCL), teat canal diameter at the proximal end of the teat canal (TCDPROX), teat canal diameter at the midpoint between the proximal and distal ends of the teat canal (TCDMID), teat canal diameter at the distal end of the teat canal (TCDDIS), teat end diameter at the midpoint between the proximal and distal ends of the teat canal (TMD), and teat canal cross-sectional area (TCLAREA). An SOP was developed, reviewed, and modified by 3 operators. Measurements were performed by the same 3 operators using an open source software program. To assess the effect of implemented modifications and the precision of ultrasound-based measurements, concordance correlation coefficients (CCC) were calculated to determine interoperator reproducibility and intraoperator repeatability. Through modifications in the SOP, interoperator CCC increased from 0.45 to 0.86 for TCL, from 0.14 to 0.66 for TCDPROX, from 0.24 to 0.66 for TCDMID, from 0.06 to 0.56 for TCDDIS, from 0.64 to 0.91 for TMD, and from 0.17 to 0.64 for TCLAREA. Intraoperator CCC over all operators were 0.91 for TCL, 0.73 for TCDPROX, 0.80 for TCDMID, 0.69 for TCDDIS, 0.94 for TMD, and 0.75 for TCLAREA. Our results indicate that measurements of teat canal dimensions can be conducted with satisfactory precision when following
\end{abstract}

Received June 13, 2017.

Accepted October 5, 2017.

${ }^{1}$ Corresponding author: mjw248@cornell.edu an SOP. Development and strict implementation of an SOP has the potential to decrease inadvertent variability of ultrasound-based measurements among operators for ultrasonographic assessment of teat dimensions.

Key words: teat canal, repeatability, reproducibility, ultrasound

\section{Technical Note}

The teat canal represents the first line of defense against mastitis-causing pathogens entering the mammary gland (O'Shea, 1987). Its anatomical structure and dimensions have therefore been the subject of multiple studies using invasive techniques, such as a modified cannula (Grindal et al., 1991; Lacy-Hulbert and Hillerton, 1995) and contrast radiography (McDonald, 1968a,b, 1975). Ultrasonography (USG) has attained increased popularity in bovine medicine within the last decades (Buczinski and O'Connor, 2016). Because of its noninvasive nature, it is perceived as a valuable diagnostic tool for clinical applications in individual cows (Braun, 2016a,b,c) and production medicine (Bollwein et al., 2016; Ollivett and Buczinski, 2016; Teixeira et al., 2017). Due to permanent improvements in ultrasound technology, some authors described the ultrasonographic appearance of the bovine teat canal (Franz et al., 2001). Subsequently, associations between teat canal dimensions as assessed with USG and cow characteristics, risk of mastitis (Klein et al., 2005), and milking technique (conventional vs. automatic milking; Khol et al., 2006) have been investigated. A further application of USG of teat canal dimensions could be examining the influence of machine milking on teat canal diameter as a measure of teat canal penetrability.

The teat canal is a delicate structure only several millimeters long (11-17 $\mathrm{mm}$ ) and a few millimeters wide (1.0-3.3 mm; Weiss et al., 2004; Klein et al., 2005; Khol et al., 2006). These small dimensions make it difficult to obtain reliable ultrasound-based measurements that represent differences between associated factors rather 
than measurement error. To make valid inferences from studies incorporating ultrasound-based measurements (i.e., teat canal dimensions), measuring techniques require high precision (interoperator reproducibility and intraoperator repeatability). Therefore, the objectives of this study were to (1) develop a standard operating procedure (SOP) for ultrasound-based measurements of teats in dairy cows that ensures low variability when measuring relevant teat dimensions and (2) determine its interoperator reproducibility and intraoperator repeatability.

This study was conducted in January and February 2017 at the Teaching Dairy Barn of the College of Veterinary Medicine, Cornell University (Ithaca, NY). All procedures were approved by the Cornell University Institutional Animal Care and Use Committee. Holstein cows $(\mathrm{n}=123)$ were housed in 2 freestall pens with sand bedding and fed a TMR that met or exceeded the National Research Council (2001) requirements. Cows were milked 3 times daily (0400, 1100, and $1900 \mathrm{~h}$ ) in a $2 \times 10$ parallel milking parlor (P2100, DeLaval International AB, Tumba, Sweden). To improve ease of ultrasonographic imaging and minimize potential confounding of teat dimensions by peripartal udder edema, inflammation, or injury, cows with the following inclusion criteria were eligible for enrollment: $\geq 30$ DIM, free of clinical mastitis for the last $4 \mathrm{wk}$, and no udder abnormalities such as nonlactating quarters or teat injuries. A convenience sample of 16 cows was selected from a list of 80 eligible cows. Cows were in their first $(\mathrm{n}=2)$, second $(\mathrm{n}=6)$, third $(\mathrm{n}=3)$, fourth $(\mathrm{n}=$ $2)$, fifth $(n=2)$, or sixth $(n=1)$ lactation and between 42 and 392 DIM. The average (mean \pm SD) previouslactation 305-d mature equivalent milk yield was 13,917 $\pm 2,383 \mathrm{~kg}$ and ranged from 9,081 to $17,622 \mathrm{~kg}$.

Teat USG of the left front and right hind teats was performed by 1 trained investigator with a portable ultrasound device (Sonosite Edge, Sonosite Inc., Bothwell, WA) and a 5- to $10-\mathrm{MHz}$ linear array transducer (L52, Sonosite Inc.). Teat scans were taken $90 \mathrm{~s}$ after initiation of premilking udder preparation immediately before milking unit attachment and within $90 \mathrm{~s}$ after milking unit detachment in the milking parlor. To avoid teat deformation, teats were immersed in warm $\left(30^{\circ} \mathrm{C}\right) \quad 0.5 \%$ chlorhexidine solution (Vet One, Boise, ID) as described by Bruckmaier and Blum (1992). To minimize interference with the dairy's milking routine, ultrasonographic scanning was performed during 3 evening $(1900 \mathrm{~h})$ milking sessions $(5,5$, and 6 cows, respectively). During teat scanning the entire width of the teat canal was screened from one side to the other by carefully moving the container on a lateromedial line, aiming for the widest diameter. When a scan of the teat was achieved with all structures clearly visible, an image was stored. All teat scans were stored on the integrated flash drive in JPEG format and transferred to a computer with a USB for image processing.

Images were evaluated visually in Windows Photo Viewer (Microsoft Corp., Redmond, WA) and classified by the first author into 1 of 3 categories as follows: good (all boundaries clear and distinct), moderate (1 blurry boundary), or poor (more than 1 blurry boundary). Only images classified as good $(\mathrm{n}=47)$ or moderate (n $=13$ ) were considered for inclusion. Measurements were performed by 3 independent operators using an open source software program (ImageJ, National Institutes of Health, Bethesda, MD). Teat dimensions measured were teat canal length (TCL), teat canal diameter at the proximal end of the teat canal (TCDPROX), teat canal diameter at the midpoint between the proximal and distal ends of the teat canal (TCDMID), teat canal diameter at the distal end of the teat canal (TCDDIS), teat end diameter at the midpoint between the proximal and distal ends of the teat canal (TMD), and teat canal cross-sectional area (TCLAREA). An SOP was developed and evaluated by the 3 operators in 2 separate trials in the following manner. Based on information compiled from the scientific literature referring to measurements of teat (canal) dimensions, a draft SOP was written.

In a 3 -h training session, the 3 operators conducted the measurements together using the draft SOP in a total of 10 images that were derived from the same herd using the procedure described herein. When disagreement in the identification of teat dimensions occurred, the 3 operators reviewed the definitions of respective boundaries until agreement was reached, and the draft SOP was modified accordingly.

In trial 1 , the modified draft SOP was evaluated by assessment of interoperator reproducibility (agreement between measurements performed by different operators). For this purpose, 25 images were randomly selected with the random number function in Microsoft Excel (2013 version, Microsoft Corp.) from a total of 60 eligible images. Identification number, teat position, and time point of teat scanning were removed. Consequently, the images were assigned a random number. Measurements were performed independently by the 3 operators using the modified draft SOP within 1 wk.

After trial 1, the modified draft SOP was reviewed by the 3 operators, possible sources of inadvertent variability (i.e., difficulties and discrepancies in the identification of boundaries) were discussed, and adjustments were implemented. Criteria that were added after trial 1 and a detailed description of the final SOP are provided in the supplemental materials. Supplemental Figure S1 (https://doi.org/10.3168/jds.2017-13326) illustrates measured teat dimensions. 
In trial 2, the final SOP was evaluated by assessment of interoperator reproducibility and intraoperator repeatability (agreement between measurements performed by the same operator). For this purpose, another 25 images were randomly selected from the remaining 35 images, duplicated, and processed as described above. Measurements of the resulting $50 \mathrm{im}-$ ages were performed independently by the 3 operators within $10 \mathrm{~d}$.

Statistical analyses were performed using Microsoft Excel (2013, Microsoft Corp.) and R Statistical Software (R Core Team, 2015). Interoperator reproducibility was assessed by calculating mean coefficients of variation $(\mathbf{C V})$ and concordance correlation coefficients (CCC) for nonrepeated measurements as described by Carrasco et al. (2013) using the cccrm package (Carrasco and Martinez, 2015) in R. Intraoperator repeatability of duplicate measurements in trial 2 was evaluated by calculating mean $\mathrm{CV}$ and $\mathrm{CCC}$ for nonlongitudinal repeated measurements as described by Carrasco et al. (2013) with the cccrm package (Carrasco and Martinez, 2015). Results of CCC were classified as poor $(<0.40)$, fair (0.40-0.59), good (0.60-0.74), and excellent (0.75-1.00) agreement according to Cicchetti (1994).

Furthermore, general linear mixed models were generated to determine differences between operators (interoperator reproducibility) and duplicate measurements (intraoperator repeatability) for each teat dimension with the nlme package in $\mathrm{R}$ (Pinheiro et al., 2016) for trial 2. To account for dependence of images among different operators, image was included as a random effect. The covariance of repeated (duplicate) measurements was modeled with the first-order autoregressive covariance structure. Operator and duplicate measurement were included as fixed effects. The $F$-tests for type III ANOVA were calculated using the car package (Fox and Weisberg, 2011). Tukey-Kramer's post hoc test was used for comparison of means between operators using the lsmeans package (Lenth, 2016).

Overall (trials 1 and 2$)$ mean $( \pm \mathrm{SD})$ values of teat dimensions were as follows: TCL, $12.67 \pm 1.96 \mathrm{~mm}$; TCDPROX, $2.45 \pm 0.50 \mathrm{~mm}$; TCDMID, $2.44 \pm 0.46$ $\mathrm{mm}$; TCDDIS, $2.42 \pm 0.43 \mathrm{~mm}$; TMD, $18.01 \pm 1.57$ $\mathrm{mm}$; and TCLAREA, $30.49 \pm 6.55 \mathrm{~mm}^{2}$.

Mean CV and CCC and 95\% confidence intervals for assessment of interoperator reproducibility in trials 1 and 2 as well as assessment of intraoperator repeatability in trial 2 are provided in Table 1. Compared with trial 1, in trial 2 interoperator $\mathrm{CV}$ decreased for all teat dimension measurements except for TCL, for which we observed a slight increase. Interoperator CCC in trial 2 increased for all measurements in comparison with trial 1, indicating improvement of interoperator reproducibility of measurements. Based on $\mathrm{CCC}$, in- teroperator reproducibility in trial 2 was excellent for TCL and TMD; good for TCDPROX, TCDMID, and TCLAREA; and fair for TCDDIS. Intraoperator CV over all operators ranged from 1.1 to $6.7 \%$. Intraoperator CCC yielded good to excellent agreement between duplicate measurements for all teat dimension measurements except for TCLAREA assessed by operator 2, who yielded fair agreement. Mixed-effects ANOVA yielded differences between operators for all teat dimension measurements $(P<0.0001)$, whereas no differences were observed between duplicate measurements within operators $(P \geq 0.19$; Table 1$)$.

Our results indicate that reliable measurements of teat dimensions can be conducted following an SOP as described herein. By implementation and consequent revision of the SOP, precision of ultrasound-based measurements of teat dimensions was remarkably increased. A similar effect of an SOP was described for the measurement of udder firmness with a dynamometer, where improvement of interoperator reproducibility was observed through modifications of the SOP in 2 subsequent experiments (correlation coefficient of interoperator reproducibility increased from 0.80 to 0.94; Bertulat et al., 2012).

Among all teat dimensions investigated, TCL and TMD had the highest CCC as well as the lowest CV between operators and within duplicate measurements. When analyzing these dimensions, the boundaries were easily identifiable. The proximal and distal boundaries of the teat canal could be visualized distinctly due to the presence of the hyperechoic keratin plug running along this length. The exterior teat walls were also easily identifiable, as the hyperechoic edges of the wall were in sharp contrast to the hypoechoic solution surrounding the teat. Additionally, TCL and TMD were the 2 longest dimensions measured. Any differences in the placement of the measuring tools were minor compared with the overall magnitude of the measurements, resulting in relatively small differences. In contrast, teat canal diameter dimensions were relatively small. With measurements such as TCDPROX, TCDMID, and TCDDIS, these placement differences had a greater effect. This could have led to greater variation between operators and duplicate measurements within operators.

Furthermore, our results show that intraoperator repeatability was better than interoperator reproducibility for all measurements examined. Several reasons have to be taken into account when trying to explain the variability present among operators. The shape of the teat canal may account for some of the difficulties in acquiring consistent measurements across operators. The proximal end of the teat canal is often not a uniform cylindrical shape. Instead, it widens as it 
TECHNICAL NOTE: TEAT CANAL ULTRASONOGRAPHY

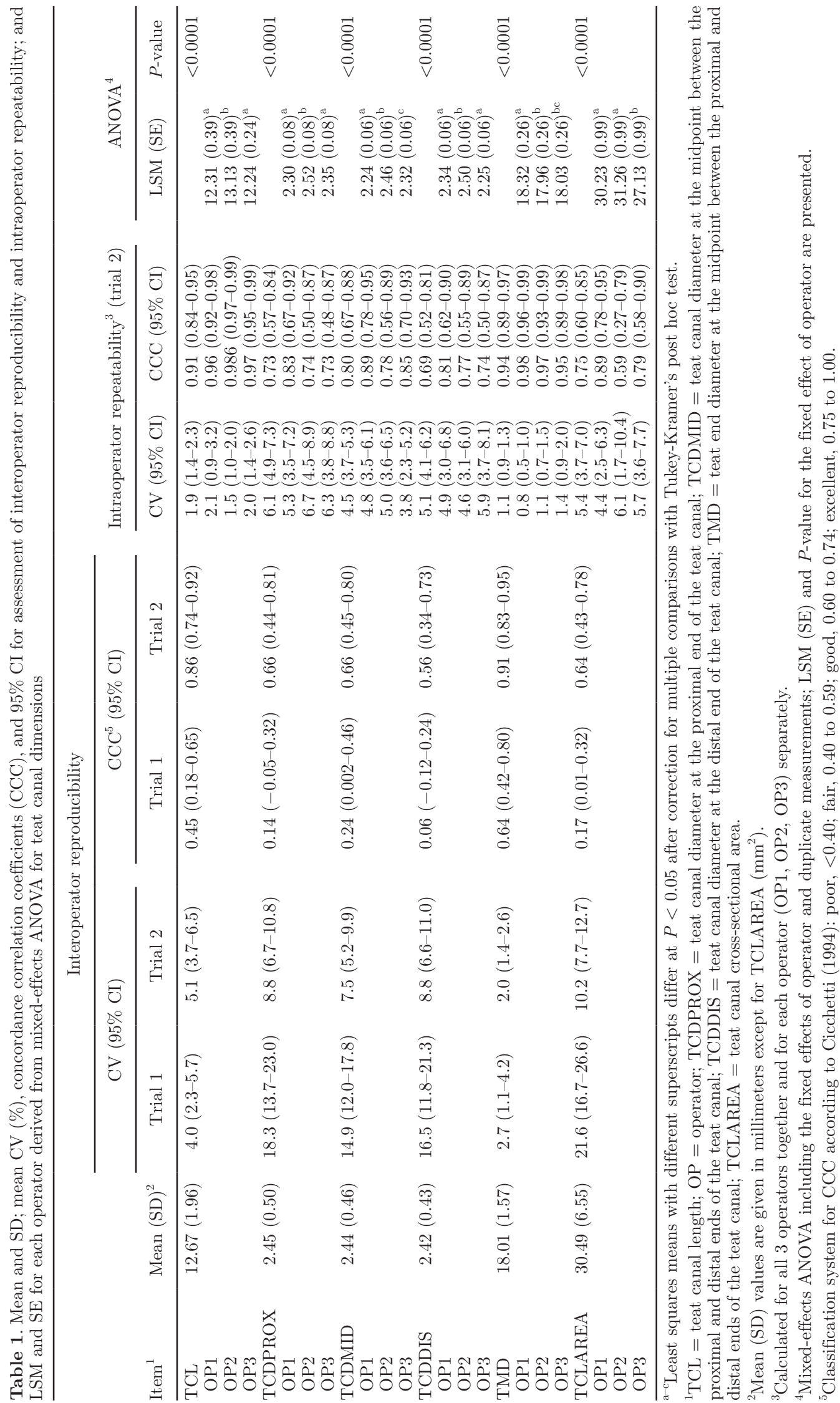


meets with the teat cistern and experiences changes in diameter and shape as it accumulates milk between the epithelial folds. The distal portion of the teat canal likewise opens up to form the teat orifice, creating a nonuniform diameter throughout the distal aspect of the teat. This may have led to difficulties in the visualization of boundaries of TCDPROX and TCDDIS, exacerbating consistent measurements among operators.

Additionally, the boundaries of the teat canal were not always clearly defined on the ultrasound images. In several cases, distinction between the hypoechoic bands of the teat canal and the more hyperechoic teat wall was challenging. This might have introduced a certain subjectivity in measuring teat canal diameter dimensions, especially in the distal and proximal regions. Inconsistencies in measuring the teat canal diameter likely contributed to differences in TCLAREA measurements.

We observed differences in repeatability of measurements within operators. Determination of CV and CCC among all investigators in a preliminary phase of a study could be used to select the most repeatable operator to minimize measurement error when analyzing images. Results of mixed-effects ANOVA identified differences in the results between operators for all teat dimension measurements. Image analysis performed by more than 1 observer may thus decrease precision in estimates and power to detect effects of interest. Although these differences were statistically significant, the interpretation should take into account their magnitude rather than the $P$-value alone, which is influenced by the number of observations in the study.

Development of an SOP should take into account all factors that contribute to variability of measurements. Such factors include (1) identification of boundaries of teat dimensions and (2) image quality (i.e., contrast, sharpness, and resolution). Future research should focus on factors that describe image quality (e.g., scanning technique) and identify a threshold for eligibility of images for evaluation.

In conclusion, TCL and TMD were the most easily reproducible measurements. Intraoperator repeatability was better than interoperator reproducibility for all teat dimension measurements investigated. Of the 3 direct measurements of the teat canal diameter, TCDMID had the greatest consistency within operators.

When studying teat canal dimensions, methods of measuring must minimize subjectivity involved in determining the teat canal boundaries and offer reproducibility and consistency. A clear description of how USG measurements were taken and reporting of all methodologies involved will help decrease inter- and intraoperator variation. This will improve reliability of data and allow better comparison between studies. De- velopment and strict implementation of an SOP has the potential to decrease information bias of ultrasoundbased measurements.

\section{ACKNOWLEDGMENTS}

This project was supported by the Clinical Fellowship Program from Cornell University, College of Veterinary Medicine (Ithaca, NY) and by the Charitable Trust of Cornell University, College of Agriculture and Life Sciences. The authors thank the staff at the Cornell Teaching Dairy Barn (Ithaca, NY). We also thank Julio O. Giordano (Department of Animal Science, Cornell University) for his invaluable technical support with the ultrasound device.

\section{REFERENCES}

Bertulat, S., C. Fischer-Tenhagen, A. Werner, and W. Heuwieser. 2012. Technical note: Validating a dynamometer for noninvasive measuring of udder firmness in dairy cows. J. Dairy Sci. 95:65506556. https://doi.org/10.3168/jds.2012-5370.

Bollwein, H., M. Heppelmann, and J. Lüttgenau. 2016. Ultrasonographic doppler use for female reproduction management. Vet. Clin. North Am. Food Anim. Pract. 32:149-164. https://doi.org/ 10.1016/j.cvfa.2015.09.005.

Braun, U. 2016a. Ascites in cattle: Ultrasonographic findings and diagnosis. Vet. Clin. North Am. Food Anim. Pract. 32:55-83. https:// doi.org/10.1016/j.cvfa.2015.09.004.

Braun, U. 2016b. Ascites in cattle: Ultrasonographic findings and diagnosis. Vet. Clin. North Am. Food Anim. Pract. 32:55-83. https:// doi.org/10.1016/j.cvfa.2015.09.004.

Braun, U. 2016c. Ultrasonographic examination of the reticulum, rumen, omasum, abomasum, and liver in calves. Vet. Clin. North Am. Food Anim. Pract. 32:85-107. https://doi.org/10.1016/j.cvfa .2015.09.011.

Bruckmaier, R. M., and J. W. Blum. 1992. B-mode ultrasonography of mammary glands of cows, goats and sheep during alpha- and beta-adrenergic agonist and oxytocin administration. J. Dairy Res. 59:151-159.

Buczinski, S., and A. M. O'Connor. 2016. Specific challenges in conducting and reporting studies on the diagnostic accuracy of ultrasonography in bovine medicine. Vet. Clin. North Am. Food Anim. Pract. 32:1-18. https://doi.org/10.1016/j.cvfa.2015.09.009.

Carrasco, J. L., and J. P. Martinez. 2015. cccrm: Concordance Correlation Coefficient for Repeated (and Non-Repeated) Measures. $\mathrm{R}$ package version 1.2.1. Accessed Feb. 26, 2017. https://CRAN.R -project.org $/$ package $=$ cccrm.

Carrasco, J. L., B. R. Phillips, J. Puig-Martinez, T. S. King, and V. M. Chinchilli. 2013. Estimation of the concordance correlation coefficient for repeated measures using SAS and R. Comput. Methods Programs Biomed. 109:293-304. https://doi.org/10.1016/j.cmpb .2012.09.002.

Cicchetti, D. V. 1994. Guidelines, criteria, and rules of thumb for evaluating normed and standardized assessment instruments in psychology. Psychol. Assess. 6:284-290. https://doi.org/10.1037/ 1040-3590.6.4.284.

Fox, J., and S. Weisberg. 2011. An R Companion to Applied Regression. 2nd ed. Sage, Thousand Oaks, CA.

Franz, S., M. Hofmann-Parisot, W. Baumgartner, G. Windischbauer, A. Suchy, and B. Bauder. 2001. Ultrasonography of the teat canal in cows and sheep. Vet. Rec. 149:109-112.

Grindal, R. J., A. W. Walton, and J. E. Hillerton. 1991. Influence of milk flow rate and streak canal length on new intramammary infection in dairy cows. J. Dairy Res. 58:383-388. 
Khol, J. L., S. Franz, D. Klein, D. Lexer, S. Waiblinger, K. Luger, and W. Baumgartner. 2006. Influence of milking technique and lactation on the bovine teat by means of ultrasonographic examination. Berl. Munch. Tierarztl. Wochenschr. 119:68-73.

Klein, D., M. Flock, J. L. Khol, S. Franz, H. P. Stuger, and W. Baumgartner. 2005. Ultrasonographic measurement of the bovine teat: Breed differences, and the significance of the measurements for udder health. J. Dairy Res. 72:296-302. https://doi.org/10 $.1017 /$ S0022029905000920.

Lacy-Hulbert, S. J., and J. E. Hillerton. 1995. Physical characteristics of the bovine teat canal and their influence on susceptibility to streptococcal infection. J. Dairy Res. 62:395-404.

Lenth, R. V. 2016. Least-Squares Means: The R Package lsmeans. J. Stat. Softw. 69:1-33. https://doi.org/10.18637/jss.v069.i0.

McDonald, J. S. 1968a. Radiographic method for anatomic study of the teat canal: Changes with lactation age. Am. J. Vet. Res. 29:1207-1210.

McDonald, J. S. 1968b. Radiographic method for anatomic study of the teat canal: Observations on 22 lactating dairy cows. Am. J. Vet. Res. 29:1315-1319.

McDonald, J. S. 1975. Radiographic method for anatomic study of the teat canal: Characteristics related to resistance to new intramammary infection during lactation and the early dry period. Cornell Vet. 65:492-499.
National Research Council. 2001. Nutrient Requirements of Dairy Cattle. 7th rev. ed. Natl. Acad. Press, Washington, DC.

O'Shea, J. 1987. Machine milking and mastitis. Section 2: Machine milking factors affecting mastitis. A literature review. Bull. Int. Dairy Fed. 215:5-32.

Ollivett, T. L., and S. Buczinski. 2016. On-farm use of ultrasonography for bovine respiratory disease. Vet. Clin. North Am. Food Anim. Pract. 32:19-35. https://doi.org/10.1016/j.cvfa.2015.09.001.

Pinheiro, J., D. Bates, S. DebRoy, D. Sarkar, and R Core Team. 2016. nlme: Linear and Nonlinear Mixed Effects Models. R package version 3.1-128. Accessed Feb. 26, 2017. https://CRAN.Rproject.org/ package $=$ nlme.

R Core Team. 2015. R: A Language and Environment for Statistical Computing. R Foundation for Statistical Computing, Vienna, Austria.

Teixeira, A. G., J. A. McArt, and R. C. Bicalho. 2017. Thoracic ultrasound assessment of lung consolidation at weaning in Holstein dairy heifers: Reproductive performance and survival. J. Dairy Sci. 100:2985-2991. https://doi.org/10.3168/jds.2016-12016.

Weiss, D., M. Weinfurtner, and R. M. Bruckmaier. 2004. Teat anatomy and its relationship with quarter and udder milk flow characteristics in dairy cows. J. Dairy Sci. 87:3280-3289. https://doi.org/ 10.3168/jds.S0022-0302(04)73464-5. 